\title{
A Bio-inspired Plume Tracking Algorithm for Mobile Sensing Swarms in Turbulent Flow
}

\author{
Dongsik Chang, Wencen Wu, Donald R. Webster, Marc J. Weissburg, and Fumin Zhang
}

\begin{abstract}
We develop a plume tracking algorithm for a swarm of mobile sensing agents in turbulent flow. Inspired by blue crabs, we propose a stochastic model for plume spikes based on the Poisson counting process, which captures the turbulent characteristic of plumes. We then propose an approach to estimate the parameters of the spike model, and transform the turbulent plume field detected by sensing agents into a smoother scalar field that shares the same source with the plume field. This transformation allows us to design path planning algorithms for mobile sensing agents in the smoother field instead of in the turbulent plume field. Inspired by the source seeking behaviors of fish schools, we design a velocity controller for each mobile agent by decomposing the velocities into two perpendicular parts: the forward velocity incorporates feedback from the estimated spike parameters, and the side velocity keeps the swarm together. The combined velocity is then used to plan the path for each agent in the swarm. Theoretical justifications are provided for convergence of the agent group to the plume source. The algorithms are also demonstrated through simulations.
\end{abstract}

\section{INTRODUCTION}

Localizing gas/odor sources are of great importance in various scenarios such as finding the leaks of poisonous chemicals, searching for survivors after a disaster, and detecting fire in its early stage. To detect and track chemical sources in an unknown environment, mobile sensing agents are deployed in the field, and various approaches have been developed such as building a map of the flow field [1]-[4], gradient-based [5]-[9] and gradient-free algorithms [10].

The fluid flow environment, in which a chemical source is present, varies depending on different Reynolds numbers. Low values of Reynolds numbers indicate smooth variations in chemical concentration, which imply well-defined gradients of the chemical concentration. At medium to high Reynolds values, chemical dispersion is dominated by turbulent mixing, which produces poorly defined and timevarying gradients [11]-[13]. The turbulent flow fluctuation at large Reynolds number brings difficulties in designing control strategies for mobile sensing agents because it is

The research work is supported by ONR grants N00014-09-1-1074, and N00014-10-10712 (YIP), and NSF grants ECCS-0841195 (CAREER) and CNS-0931576

Dongsik Chang, Wencen $\mathrm{Wu}$, and Fumin Zhang are with the Schoo of Electrical and Computer Engineering, Georgia Institute of Technology, Atlanta, GA, 30332, USA \{dsfrancis3, wwencen3, fumin\}egatech . edu

Donald R. Webster is with the School of Civil and Environmental Engineering, Georgia Institute of Technology, Atlanta, GA, 30332, USA dwebsterece.gatech.edu

Marc J. Weissburg is with the School of Biology, Georgia Institute of Technology, Atlanta, GA, 30332, USA marc.weissburgabiology.gatech.edu impossible to use analytical methods or simple numerical simulations to predict the characteristics of odorant plumes [11]. To successfully navigate mobile sensing agents, various algorithms have been developed inspired by biology such as E. coli [6], [10], beetles [10], blue crab [14], [15], silkworm moth [7], [16]-[18], and bees [19].

Inspired by behaviors of blue crabs, Webster et al. [15] develop and implement a plume tracking algorithm in a controlled turbulent flow environment. They develop a signal processing strategy that is able to replicate behavioral responses of blue crabs tracking a chemical stimuli. To answer the challenge that the characteristics of the turbulent flow is difficult to measure, they install sensor arrays on an experimental vehicle to detect plume spikes and estimate frequencies of the detected plume spikes. The spike information is then processed by the tracking system to guide the upstream and cross-stream motions of the vehicle so that it can move towards and declare the plume source.

Inspired by their results, we investigate the plume tracking problem in turbulent fluid fields using mobile sensing agents. The goal is to control a swarm of mobile sensing agents to detect and track a plume source from initial locations that are downstream to the source. We investigate the mechanism of the turbulent flow field and propose a stochastic modeling method for plume spikes using Poisson processes that describes the characteristics of chemical plumes. We define Poisson counters to indicate the detection of a spike, the duration of which is implied by the rates of the Poisson counters.

For the plume tracking system, we design velocity controls for an $\mathrm{N}$-agent group to move towards a plume source. A novel design of our strategy is that instead of letting the agent group locate a plume source in a turbulent fluid field, we transform the detected turbulent flow field into a smooth scalar field, the gradient of which is well-defined, so that we can design control laws for the agent group in the smooth field. Inspired by recently observed fish behaviors [20] and our previous work on non-gradient source-seeking algorithms [21], we decompose the velocities of each agent into two parts: One part maintains the constant distance among agents, and the other part, which is proportional to the estimated duration of spikes, controls the entire group to move towards the plume source. We prove that, under the control laws, the moving direction of the agent group converges to the gradient direction of the smooth field. The computed velocities are then used by each agent to plan its path forward, which leads the group to the plume source in the turbulent flow.

The rest of the paper is organized as follows. Section 


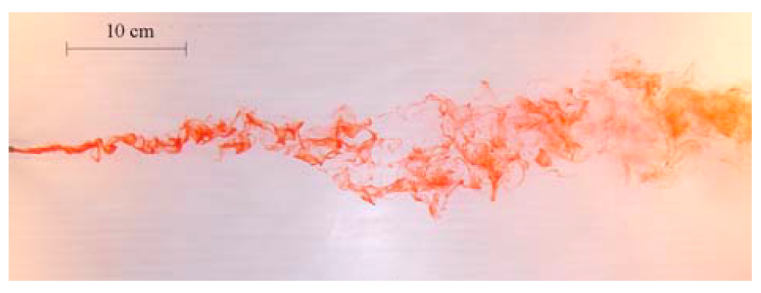

Fig. 1. Flow visualization of the chemical plume in a controlled turbulent flow.The view is from above, with the flow moving from left to right.

II introduces the modeling of chemical plume spikes using Poisson counters. Section III discusses methods for estimating spike parameters. Section IV presents control strategies for controlling two mobile robots to track a plume and the corresponding path planning algorithm. Section V demonstrates simulations results of the proposed algorithm. Section VI presents concluding remarks.

\section{Problem Formulation}

We assume a plume is generated from a single source releasing chemicals into a turbulent flow field. A swarm of mobile sensors are deployed into the fluid. Each platform is able to measure the level of chemical concentration at its current location and move freely in the fluid, overcoming the flow. The research goal of this paper is to develop a source seeking algorithm to enable the swarm to move to the source from initial locations that are downstream to the source, where plume can be measured. This problem is challenging since the chemical concentration within a plume created by a turbulent flow has very high spatial and temporal variation (see Fig. 1), therefore existing gradient based methods for smooth fields do not apply.

In [15], based on experimental data collected from plumes in turbulent field, it is shown that sensors distributed across the body of a crab are able to detect spikes in the chemical concentration. The magnitude and duration of detected spikes may have been utilized by the crab to direct its motion towards the chemical source. An algorithm has been proposed to control an experimental platform equipped with three sensors to emulate this source seeking behavior of a crab. High rate of successful source seeking behaviors have been observed from the experiments. However, the mechanism behind such success have not been revealed in [15], which prevents its application to a swarm of mobile sensors.

\section{Modeling And Detecting Spikes}

Based on the experimental data in [22], we learn that when a mobile sensor moves in a chemical plume at constant speed, the measurement of concentration along its trajectory will display spikes as shown in Fig. 2. A spike can then be detected by comparing the measurement with a given threshold. The occurrences of spikes display a random nature. Another important observation is that as the sensor gets close to the plume source, the average frequency of spike

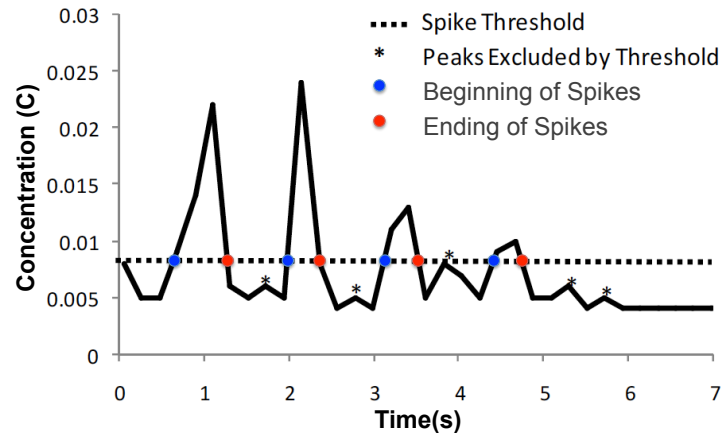

Fig. 2. Concentration measured along the trajectory of a moving sensor in a turbulent chemical plume.

arrival decreases in applying a constant threshold [22], and intuitively the duration of each spike becomes longer.

We now introduce a stochastic model to describe the random occurrences of spikes along the trajectory. First, we define the spike indicator as $s=\left\{s_{1}, s_{2}\right\}$, where $s_{1}, s_{2} \in \mathbb{Z}$. When $s=s_{1}$, there is no spike at the current position of the mobile sensor, and $s=s_{2}$ indicates otherwise. The spike indicator $s$ satisfies a stochastic differential equation driven by Poisson counters as below:

$$
d s=\left(s_{1}-s\right) d N_{12}+\left(s_{2}-s\right) d N_{21},
$$

where $d N_{12}$ is the Poisson jump process that triggers the state $s$ to jump from $s_{1}$ to $s_{2}$, and $d N_{21}$ is the Poisson jump process that triggers the state $s$ to jump from $s_{2}$ to $s_{1}$. Along the trajectory, a state jump from $s_{2}$ to $s_{1}$ indicates the beginning of a spike and a jump from $s_{1}$ back to $s_{2}$ indicates the ending of a spike. These transitions happen randomly as Poisson processes. The rates of the jump process $d N_{12}$ is $\lambda_{12}$ and the rates of $d N_{21}$ is $\lambda_{21}$, these rates determine how frequently state transitions happens. Therefore, $\lambda_{12}$ and $\lambda_{21}$ affect the duration of a spike as well as the averaged frequency of the occurrences of the random spikes.

Suppose a mobile sensor can detect spikes using the thresholding method, then the rates $\lambda_{12}$ and $\lambda_{21}$ can be inferred from the beginning and ending of consecutive spikes. Consider the current time as $t$, and suppose $j$-th spikes have been detected along the trajectory of the mobile sensor. Let us denote by $T_{j}$ and $T_{j}^{\prime}$ the beginning and ending time of the $j$-th spike, respectively. We will use the timings for spikes $j-1$ and $j$ to estimate the rate of state transition as

$$
\begin{aligned}
& \hat{\lambda}_{12}(t)=\frac{1}{T_{j}-T_{j-1}^{\prime}} \\
& \hat{\lambda}_{21}(t)=\frac{1}{T_{j}^{\prime}-T_{j}} .
\end{aligned}
$$

Equations (1) and (2) models the timing of spikes. Next, we consider the noises or imperfections in the sensor that cause variations in the measurements of the concentration. These variations will cause inaccuracy in determining the timing of the state transitions. Therefore the $\hat{\lambda}_{12}(t)$ and $\hat{\lambda}_{21}(t)$ in Eq. (2) are noisy estimates for the true values of the rates. 


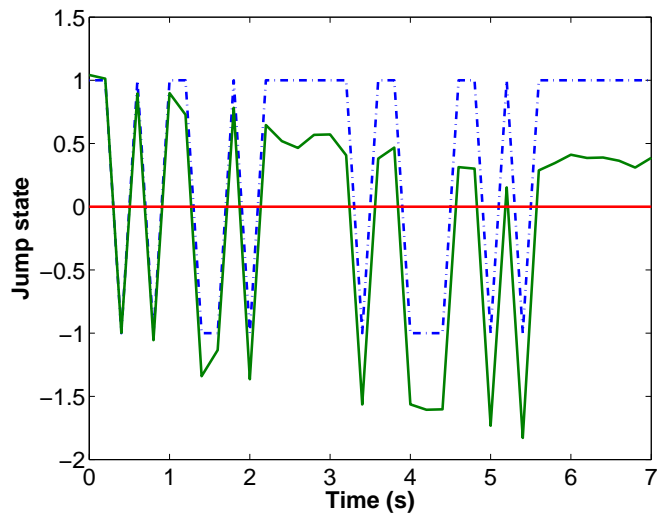

Fig. 3. Simulated timing of spikes modeled by the stochastic Poisson processes. $s_{1}=-1, s_{2}=1$, and $\sigma=0.08$. The dotted blue line indicates the true value of the state $s$ without noise. The added Gaussian noise severely corrupted the state $s$, and will cause significant uncertainties in the timing estimates.

To model the effect of sensor noises on the transition timing, we assume $s$ is also corrupted by zero mean, Gaussian noises with variance $\sigma$. Fig. 3 illustrates a case when $s_{1}=-1$, $s_{2}=1$, and $\sigma=0.08$.

We will later design controllers based on the noisy estimates of the rates $\hat{\lambda}_{12}(t)$ and $\hat{\lambda}_{21}(t)$. However, to evaluate the performance of our controller design, we provide a ground truth for $\lambda_{12}$ and $\lambda_{21}$ that matches the results in [22]. Let $\mathbf{r} \in \mathbb{R}^{3}$ be the current location of the sensor in the field. And suppose the chemical source is located at position $\mathbf{r}_{0}$. Let the distance between the sensor and the source be $d_{p}=\left\|\mathbf{r}_{0}-\mathbf{r}\right\|$, We assume that $\lambda_{12}$ is a monotonically increasing function of the the distance $d_{p}$, and $\lambda_{21}$ is inverse proportional to the distance, e.g.,

$$
\begin{aligned}
& \lambda_{12}=k_{\lambda} \frac{1}{g\left(d_{p}\right)} \\
& \lambda_{21}=k_{\lambda}^{\prime} g\left(d_{p}\right),
\end{aligned}
$$

in which $k_{\lambda}$ and $k_{\lambda}^{\prime}$ are constants chosen by design. A simple choice of $g(\cdot)$ is to let $g\left(d_{p}\right)=d_{p}$.

Define a function

$$
f\left(d_{p}\right)=\frac{\lambda_{12}+\lambda_{21}}{\lambda_{12}}=\frac{k_{\lambda}^{\prime}}{k_{\lambda}} g\left(d_{p}\right)^{2}+1,
$$

which is a smooth function of distance $d_{p}$. Since the source is located at $d_{p}=0$, where the function $f\left(d_{p}\right)$ has a unique minimum value 1 , the problem of finding the plume source in the turbulent field is now equivalent to finding the minimum point in the smooth field $f\left(d_{p}\right)$. The only challenge here is that the function $f\left(d_{p}\right)$ has to be estimated from the sensor measurements. Using the estimates $\hat{\lambda}_{12}(t)$ and $\hat{\lambda}_{21}(t)$, the mobile sensor can compute a noisy estimate of $f\left(d_{p}\right)$ as

$$
\hat{f}\left(d_{p}\right)=\frac{\hat{\lambda}_{12}+\hat{\lambda}_{21}}{\hat{\lambda}_{12}} .
$$

Therefore, through stochastic modeling, we have converted a source seeking problem within a turbulent field into a

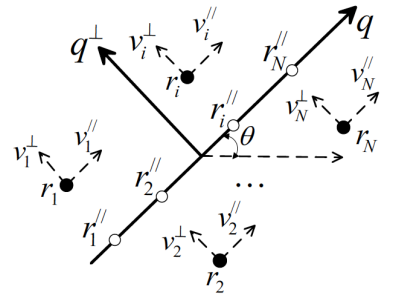

Fig. 4. Decomposition of the velocities of the agents in a $\mathrm{N}$-agent group.

minimum seeking problem in a smooth field corrupted by non-Gaussian noises.

\section{Plume Tracking Control}

In this section, we design control strategies for a swarm of mobile sensing agents to locate a plume source based on the estimated plume spike parameters. Our strategy is inspired by recent observations of fish behaviors that fish speeds up when the light intensity increases, and slows down when the light intensity decreases so that the fish school is able to converge to the darkest area in a light field [20].

Suppose $N$ sensing agents are deployed in a plume. Let $\mathbf{r}_{i}$ represent the position of the $i$-th agent and $\mathbf{r}_{c}$ represent the center of the group. Then, we derive $\mathbf{r}_{c}=\frac{1}{N} \sum_{i=1}^{N} \mathbf{r}_{i}$. As introduced previously, for $i=1,2, \ldots, N$, let $d_{p, i}$ be the distance between the plume source and the $i$-th sensing agent. Without loss of generality, let us assume the plume source is located at position $\mathbf{r}_{0}=(0,0)$. Therefore, $d_{p, i}=\left\|\mathbf{r}_{i}\right\|$.

\section{A. Determining Velocities of the Agents}

Define the inertial frame as $X_{I}$ and $Y_{I}$. Arbitrarily select a baseline $\mathbf{q}$ as an unit vector that forms an angle $\theta$ with the inertial frame $X_{I}$. Define $\mathbf{q}^{\perp}$ to be the vector perpendicular to $\mathbf{q}$ that forms a right handed frame with $\mathbf{q} . \mathbf{q}$ and $\mathbf{q}^{\perp}$ intersects at $\mathbf{r}_{c}$. As illustrated in Fig. 4, for each agent, we decompose its velocity into two parts [21]: $\mathbf{v}_{i}^{\perp}$, which is perpendicular to $\mathbf{q}$, and $\mathbf{v}_{i}^{/ /}$, which is aligned with $\mathbf{q}$ and maintains formation. Then, $\mathbf{v}_{i}=\mathbf{v}_{i}^{\perp}+\mathbf{v}_{i}^{/ /}$. For $\mathbf{v}_{i}^{\perp}=v_{i}^{\perp}\left(\begin{array}{c}-\sin \theta \\ \cos \theta\end{array}\right)$, we design $v_{i}^{\perp}, i=1,2$, as

$$
v_{i}^{\perp}=K \hat{f}\left(d_{p, i}\right)+C,
$$

where $K$ and $C$ are constants selected by design.

Along direction $\mathbf{q}$, let $\mathbf{r}_{i}^{/ /}$be the projection of location $\mathbf{r}_{i}$ onto vector $\mathbf{q}$, as illustrated in Fig. 4. For agent $i$, we define set $\mathscr{N}_{i}$ to contain the closest agents to agent $i$ to the right and to the left along direction q. For example, as shown in Fig. 4, $\mathscr{N}_{1}=\{2\}, \mathscr{N}_{i}=\{i-1, i+1\}, i \neq 1, N$, and $\mathscr{N}_{N}=\{N-1\}$. The goal is to design $\mathbf{v}_{i}^{/ /}$so that the relative distance from $\mathbf{r}_{i}^{/ /}$to $\mathbf{r}_{j}^{/ /}, i \neq j$, converges to a constant $a_{i j}^{0}$. Furthermore, we require that $\mathbf{v}_{c}^{/ /}=\frac{1}{N} \sum_{i=1}^{N} \mathbf{v}_{i}^{/ /}=0$. Therefore, for $\mathbf{v}_{i}^{/ /}=$ $v_{i}^{/ /}\left(\begin{array}{c}\cos \theta \\ \sin \theta\end{array}\right)$, we $\operatorname{design} v_{i}^{/ /}$as

$$
v_{i}^{/ /}=k_{p} \sum_{j \in \mathscr{N}_{i}}\left(\left(\mathbf{r}_{j}-\mathbf{r}_{i}\right) \cdot \mathbf{q}-a_{j, i}^{0}\right),
$$


where $a_{i, j}^{0}=-a_{j, i}^{0}$. We can prove that, under the formation control law (7), the relative distance between every two agents converges to a constant [21]. Given $v_{i}^{\perp}$ and $v_{i}^{/ /}$, each agent now has its velocity given by

$$
\begin{aligned}
\mathbf{v}_{i} & =\left(K \hat{f}\left(d_{p, i}\right)+C\right)\left(\begin{array}{c}
-\sin \theta \\
\cos \theta
\end{array}\right) \\
& +k_{p} \sum_{j \in \mathscr{N}_{i}}\left(\left(\mathbf{r}_{j}-\mathbf{r}_{i}\right) \cdot \mathbf{q}-a_{j, i}^{0}\right)\left(\begin{array}{c}
\cos \theta \\
\sin \theta
\end{array}\right),
\end{aligned}
$$

and $\mathbf{v}_{c}^{/ /}=\frac{1}{N} \sum_{i=1}^{N} \mathbf{v}_{i}^{/ /}=0$, we obtain the velocity of the formation center as

$$
\mathbf{v}_{c}=\left(\frac{1}{N} K \sum_{i=1}^{N} \hat{f}\left(d_{p, i}\right)+C\right)\left(\begin{array}{c}
-\sin \theta \\
\cos \theta
\end{array}\right) .
$$

If we arbitrarily choose the baseline $\mathbf{q}=\frac{\mathbf{r}_{i}-\mathbf{r}_{j}}{\left\|\mathbf{r}_{i}-\mathbf{r}_{j}\right\|}$, in which $i \neq j$, then the angular velocity of the group can be calculated as

$$
\dot{\theta}=\omega=\frac{v_{i}^{\perp}-v_{j}^{\perp}}{\left\|\mathbf{r}_{i}-\mathbf{r}_{j}\right\|}=\frac{K}{\left\|\mathbf{r}_{i}-\mathbf{r}_{j}\right\|}\left(\hat{f}\left(d_{p, i}\right)-\hat{f}\left(d_{p, j}\right)\right) .
$$

Since the noise in the estimation of $\hat{f}\left(d_{p, i}\right)$ is unstructured and complex, we use $\hat{f}\left(d_{p, i}\right)=f\left(d_{p, i}\right)$ to analyze the convergence of the moving direction of the group to gradient directions. If we use Taylor expansion to approximate $f\left(d_{p, i}\right)$, we have

$$
f\left(d_{p, i}\right)=f\left(d_{p, c}\right)+\nabla f\left(d_{p, c}\right) \cdot\left(\mathbf{r}_{i}-\mathbf{r}_{c}\right)+\text { H.O.T }
$$

where $d_{p, c}$ is the distance from the formation center to the plume source, $\nabla f\left(d_{p, c}\right)$ is the gradient of $f\left(d_{p, c}\right)$ at $d_{p, c}$, and H.O.T represents higher order terms in the above Taylor expansion. Denote the angle between the gradient direction $\nabla f\left(d_{p, c}\right)$ and the inertial frame $X_{I}$ as $\alpha \in[-\pi, \pi]$. Then, we derive from Equation (10) that

$$
\begin{aligned}
\dot{\theta} & \cong \frac{K}{\left\|\mathbf{r}_{i}-\mathbf{r}_{j}\right\|}\left(\nabla f\left(d_{p, c}\right) \cdot\left(\mathbf{r}_{i}-\mathbf{r}_{j}\right)\right)=K\left(\nabla f\left(d_{p, c}\right) \cdot \mathbf{q}\right) \\
& =K\left\|\nabla f\left(d_{p, c}\right)\right\|\left(\frac{\nabla f\left(d_{p, c}\right)}{\left\|\nabla f\left(d_{p, c}\right)\right\|} \cdot \mathbf{q}\right) \\
& =-K\left\|\nabla f\left(d_{p, c}\right)\right\| \sin \left(\theta-\alpha-\frac{\pi}{2}\right)
\end{aligned}
$$

Choose the state to be $\theta-\alpha$, then we obtain

$$
\dot{\theta}-\dot{\alpha}=-K\left\|\nabla f\left(d_{p, c}\right)\right\| \sin \left(\theta-\alpha-\frac{\pi}{2}\right)-\dot{\alpha} .
$$

When $\left\|\nabla f\left(d_{p, c}\right)\right\| \neq 0$, the above system has a stable equilibrium $\theta-\alpha=\frac{\pi}{2}$ and an unstable equilibrium $\theta-\alpha=$ $-\frac{\pi}{2}$. Given the above system, we introduce the following proposition stating that the agent group will move towards the plume source.

Proposition 4.1: If the gradient direction $\alpha$ is constant, that is, $\dot{\alpha}=0$, then, as $t \rightarrow \infty, \lim _{t \rightarrow \infty} \theta(t)=\alpha+\frac{\pi}{2}$. If the rate of change $\dot{\alpha} \neq 0$ is considered as an input to the system (13), then $\theta-\alpha=\frac{\pi}{2}$ is an equilibrium of (13) that is inputto-state stable (ISS).

\section{Proof:}

If $\dot{\alpha}=0$, we choose a Lyapunov candidate function as

$$
V=-\ln \left(\cos \left(\frac{\theta-\alpha-\frac{\pi}{2}}{2}\right)\right)
$$

We calculate

$$
\begin{aligned}
\dot{V} & =\tan \left(\frac{\theta-\alpha-\frac{\pi}{2}}{2}\right)(\dot{\theta}-\dot{\alpha}) \\
& =-2 K\left\|\nabla f\left(d_{p, c}\right)\right\| \sin ^{2}\left(\frac{\theta-\alpha-\frac{\pi}{2}}{2}\right)-\tan \left(\frac{\theta-\alpha-\frac{\pi}{2}}{2}\right) \dot{\alpha} \\
& =-2 K(1-\varepsilon)\left\|\nabla f\left(d_{p, c}\right)\right\| \sin ^{2}\left(\frac{\theta-\alpha-\frac{\pi}{2}}{2}\right) \\
& -2 K \varepsilon\left\|\nabla f\left(d_{p, c}\right)\right\| \sin ^{2}\left(\frac{\theta-\alpha-\frac{\pi}{2}}{2}\right)-\tan \left(\frac{\theta-\alpha-\frac{\pi}{2}}{2}\right) \dot{\alpha} \\
& \leq-2 K(1-\varepsilon)\left\|\nabla f\left(d_{p, c}\right)\right\| \sin ^{2}\left(\frac{\theta-\alpha-\frac{\pi}{2}}{2}\right),
\end{aligned}
$$

when $|\dot{\alpha}| \leq K \varepsilon\left\|\nabla f\left(d_{p, c}\right)\right\|\left|\sin \left(\theta-\alpha-\frac{\pi}{2}\right)\right|$ and $0<\varepsilon<1$. Therefore, according to Theorem 4.19 in [23], if $\dot{\alpha}$ is considered as the input, the system (13) is input-to-state stable (ISS). If the input $\dot{\alpha}=0, \theta$ converges to the equilibrium point $\alpha+\frac{\pi}{2}$. If the rate of change $\dot{\alpha}$ is bounded, then at the steady state, the deviation $\left|\left(\theta-\alpha-\frac{\pi}{2}\right)\right|$ is also bounded. Therefore, as $t \rightarrow \infty, \lim _{t \rightarrow \infty} \theta(t)=\alpha+\frac{\pi}{2}$, which indicates that the agent group will move towards the minimum of field $f\left(d_{p, c}\right)$.

\section{B. Path Planning}

Proposition 4.1 indicates that, if we use Eq. (8) as feedback control for the velocities of the agents in continuous time, the moving direction of the agent group will converge to the gradient direction of field $f\left(d_{p}\right)$. However, in our simulation, we do not control the velocities of the agents in continuous time. We discretize the system and use the designed velocities for the path planning of the agent group. Denote $\mathbf{r}_{i, k}$ as the position of the $i$-th sensing agent at time instant $t_{k}$. We propose the following path-planning algorithm.

Algorithm 1: Repeat the the following steps for $i=$ $1,2, \ldots, N$. At location $\mathbf{r}_{i, k}$, the $i$-th sensing agent

1) takes measurements of the turbulent field values for a finite time $T$, called the "waiting time".

2) estimates $\hat{\lambda}_{12}$ and $\hat{\lambda}_{21}$ based on the measurements, estimates $\hat{f}\left(d_{p}\right)$ based on Eq. (5), and using Eq. (8) determines the velocity $\mathbf{v}_{i, k}$, which generates a planned trajectory.

3) moves forward along the trajectory over a finite motion horizon time $\tau$ so that $\mathbf{r}_{i, k+1}=\mathbf{r}_{i, k}+\tau \mathbf{v}_{i, k}$.

Let $k=k+1$ and repeat the above steps until the function $\hat{f}\left(d_{p, i}\right)$ estimated by an agent is sufficiently close to 1 , indicating the vicinity of the source.

We will show in the simulation section that by using the speed control of (6) and (7) and the path planning algorithm 1 , a two-agent group is able to move towards the plume source while maintaining a constant formation. 


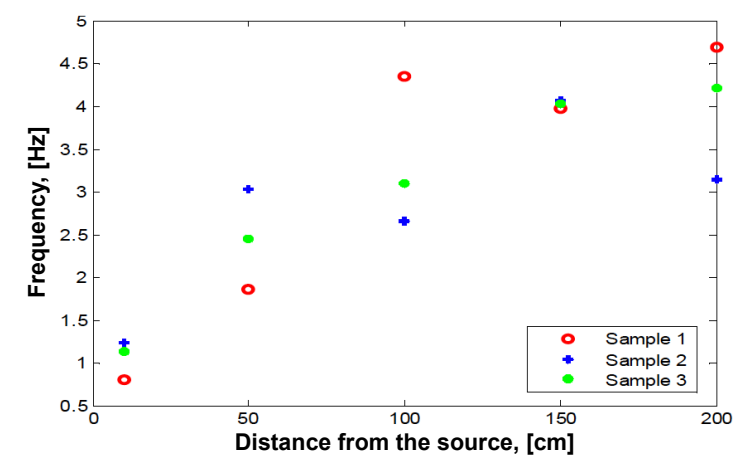

Fig. 5. Frequency of concentration spikes $[\mathrm{Hz}]$ as a function of distance from the source.
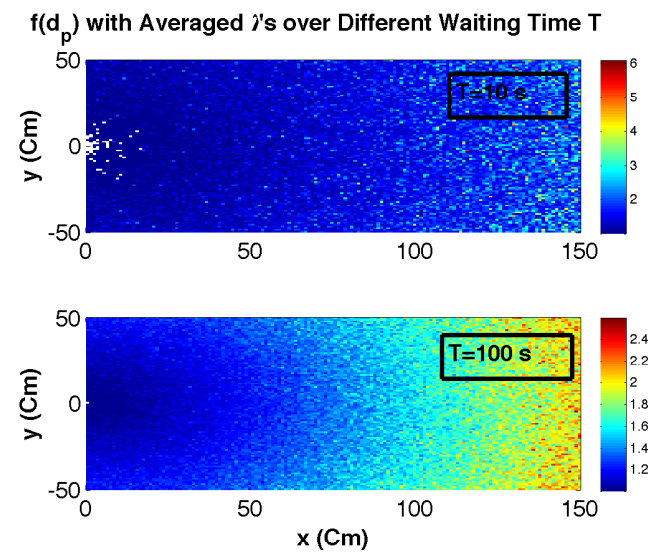

Fig. 6. An estimated field of $f\left(d_{p}\right)$ when the waiting time $T=$ $\{10,100\} s$. The plume source is located at $(0,0)$. The rates $\lambda$ 's are estimated using Eq. (2) for each time step, and then averaged over the period of waiting time.

\section{Simulation Results}

The simulation is setup by first generating a simulated two dimensional turbulent plume field that matches experimental data. The ground truth $k_{\lambda}$ and $k_{\lambda}^{\prime}$ parameters for the spikes in such a field are selected from Fig. 5 from [22]. In the figure, three spike samples are measured at each of five locations with different distances to the source. Since all three measurements taken at distance $150 \mathrm{~cm}$ have the frequency of $4 \mathrm{~Hz}$, we choose the reference frequency $f_{r}=4 \mathrm{~Hz}$ and reference distance $d_{r}=150 \mathrm{~cm}$. Then, we let $k_{\lambda}=f_{r} d_{r}$ and $k_{\lambda}^{\prime}=\frac{f_{r}}{d_{r}}$. These parameters then determine the rates for spikes $\lambda_{12}$ and $\lambda_{21}$ used in our simulation.

At each location in the plume field, we simulate a train of spikes using the stochastic model in Eq. (1). We allow a sensing agent at its current location to measure this train for the waiting time $T$ as described in Algorithm 1. Then, a noisy estimate of the field $f\left(d_{p}\right)$ will be generated by the agent. Fig. 6 plots this noisy estimate over the entire field for $T=\{10,100\} s$. When $T=100 \mathrm{~s}$, we can see that the field is close to a smooth field, but with smaller waiting time $T$, we can observe that the field becomes more noisy.

We simulate two mobile sensing agents in the simulated
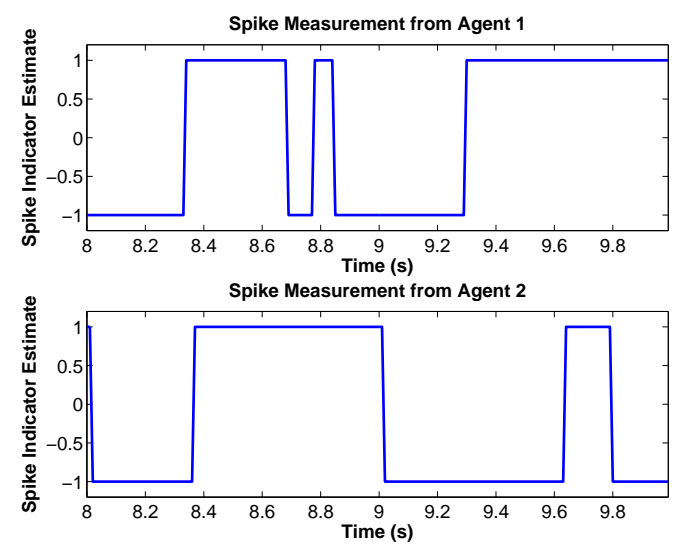

Fig. 7. Example of spikes estimated by mobile sensing agents from plume measurements with $s_{1}=-1$ and $s_{2}=1$.

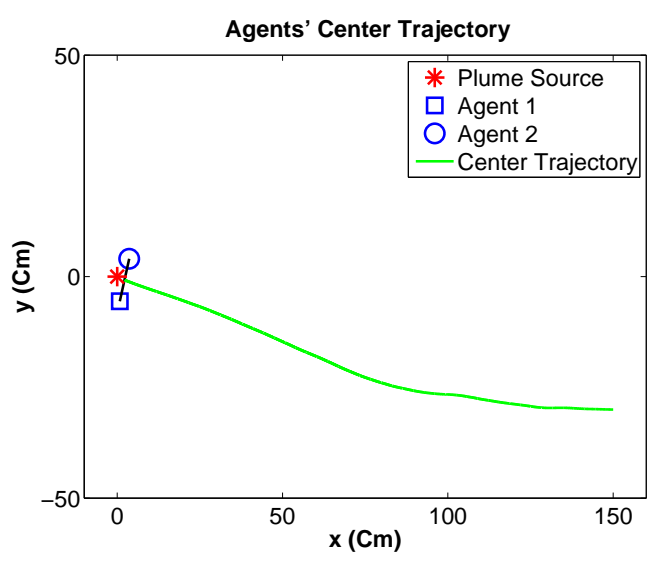

Fig. 8. The trajectory of the center of two mobile sensing agents in a plume tracking simulation. The plume source is located at $(0,0)$ and the agents are deployed at $(150,-35)$ and $(150,-25)$. The waiting time $T=100 \mathrm{~s}$ at each position and the agents move forward for $\tau=1 s$ after the velocities are determined.

turbulent fluid field. The simulated agents are initially deployed at $(150,-35)$ and $(150,-25)$, and the two agents will keep a distance $\left|a^{0}\right|=10 \mathrm{~cm}$. To enable the agents to find the plume source in the field, Algorithm 1 is applied. The agents will wait and estimate the averaged plume field for the waiting time $T$, then make a move for $\tau=1 \mathrm{~s}$. Each agent does not need the entire field information to navigate. Instead, they require the spike parameters $\hat{\lambda}_{12}, \hat{\lambda}_{21}$ only at their current positions to compute $\hat{f}\left(d_{p, i}\right)$ and $\mathbf{v}_{i}$ in the algorithm, which takes very small computational cost and hence enables real-time implementation. For the controller parameters in Eq. (8), we set $K=3, C=-3$, and $k_{p}=1$.

Results of the algorithm 1 are illustrated by Figures 7 and 8. During the waiting time, agents measure plume intensities and detect spikes as in Fig. 7. After estimating $\hat{\lambda}_{12}$ and $\hat{\lambda}_{21}$ using Eq. (2), the agents compute the velocities and move forward for $\tau=1 \mathrm{~s}$. Fig. 8 shows the trajectory (the green line) of the center of the two agents towards the source. The ending positions of the two agents are marked 


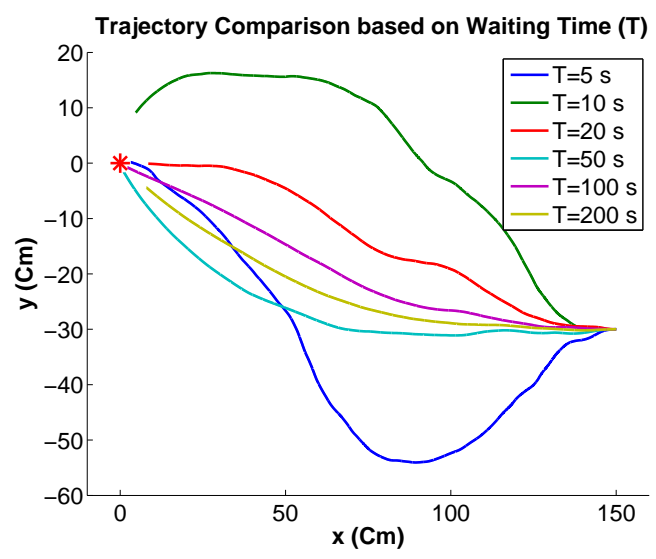

Fig. 9. Trajectory comparison of the center of two mobile sensing agents under six different choices of the waiting time $T$. Each group of agents shows different trajectories. It can be observed that longer waiting time creates shorter trajectories.

by a blue square and a blue circle, respectively. The relative displacement between the two agents is plotted as the black line connecting the two ending positions. We see that the relative distance between the agents is maintained till the end.

We have performed multiple simulations with different values of the waiting time $T$. Figure 9 shows trajectories under six values of $T: 5 s, 10 s, 20 s, 50 s 100 s$, and 200 $s$. The figure suggests that smaller waiting time generates longer trajectories. We have also observed that for smaller waiting time, the trajectories differ from trial to trial and may even not converge to the plume source. This fact is mainly because the accuracy of the estimates for the rates of spikes increases when the length of waiting time increases. However, longer waiting time implies much slower convergence rate to the source. Blue crabs are able to track roughly $2 m$ in $30 s$ or less [15], and our best performance from the trials is $150 \mathrm{~cm}$ in $105.90 \mathrm{~s}$ when waiting time is small $(T=15 s)$. We see there is still large room for improvement in our algorithm.

\section{CONCLUding REMARKS}

In this paper, we develop a plume tracking algorithm for a swarm of mobile sensing agents in turbulent plume fields. Our plume sensing strategy is inspired by blue crabs. We propose a stochastic method to model plume spikes detected by mobile sensing agents and estimate the rate parameters of the spikes. Then, we are able to transform the measured spike fields into a smooth scalar field. Our control and path planning strategy is inspired by the source seeking behaviors of fish schools. We develop a simple velocity control for the agents to achieve source seeking and also maintain the swarm. We prove the convergence of the moving direction of the agent group towards the plume source in the transformed field. Simulation results have demonstrated the effectiveness of the proposed path planning algorithm that combines the two bio-inspired strategies for sensing and navigation in a turbulent plume field.

\section{REFERENCES}

[1] J. A. Farrell, S. Pang, and W. Li, "Plume mapping via hidden Markov methods," IEEE Transactions on Systems, Man, and Cybernetics, Part B: Cybernetics, vol. 33, no. 6, pp. 850-863, 2003.

[2] A. Lilienthal and T. Duckett, "Building gas concentration gridmaps with a mobile robot," Robotics and Autonomous Systems, vol. 48, no. 1, pp. 3-16, 2004.

[3] S. Pang and J. A. Farrell, "Chemical plume source localization," IEEE Transactions on Systems, Man, and Cybernetics, Part B: Cybernetics, vol. 36, no. 5, pp. 1068-1080, 2006.

[4] G. Ferri, M. V. Jakuba, A. Mondini, V. Mattoli, B. Mazzolai, D. R. Yoerger, and P. Dario, "Mapping multiple gas/odor sources in an uncontrolled indoor environment using a Bayesian occupancy grid mapping based method," Robotics and Autonomous Systems, vol. 59, no. 11 , pp. 988-1000, 2011.

[5] S. Kazadi, R. Goodman, D. Tsikata, and H. Lin, "An autonomous water vapor plume tracking robot using passive resistive polymer sensors," Autonomous Robots, vol. 9, no. 2, pp. 175-188, 2000.

[6] C. Lytridis, G. Virk, Y. Rebour, and E. Kadar, "Odor-based navigational strategies for mobile agents," Adaptive Behavior, vol. 9, no. 3-4, pp. 171-187, 2001.

[7] J. A. Farrell, P. Shuo, and W. Li, "Chemical plume tracing via an autonomous underwater vehicle," IEEE Journal of Oceanic Engineering, vol. 30 , no. 2, pp. 428-442, 2005.

[8] A. Lilienthal and T. Duckett, "Experimental analysis of smelling braitenberg vehicles," Advanced Robotics, vol. 8, no. 8, pp. 817-834, 2004.

[9] R. A. Russell, "Robotic location of underground chemical sources," Robotica, vol. 22, pp. 109-115, 2004.

[10] R. Russella, A. Bab-Hadiashar, R. L. Shepherd, and G. G. Wallace, "A comparison of reactive robot chemotaxis algorithms," Robotics and Autonomous Systems, vol. 45, no. 2, pp. 83-97, 2003.

[11] D. R. Webster and M. J. Weissburg, "Chemosensory guidance cues in a turbulent chemical odor plume," Limnology and Oceanography, vol. 46, no. 5, pp. 1034-1047, 2001.

[12] _ - "The hydrodynamics of chemical cues among aquatic organisms," Annual Review of Fluid Mechanics, vol. 41, no. 1, pp. 73-90, 2009.

[13] G. Kowadlo and R. a. Russell, "Robot odor localization: A taxonomy and survey," The International Journal of Robotics Research, vol. 27, no. 8, pp. 869-894, 2008.

[14] M. J. Weissburg and D. B. Dusenbery, "Behavioral observations and computer simulations of blue crab movement to a chemical source in a controlled turbulent flow," Experimental Biology, vol. 205, pp. 3387-3398, 2002

[15] D. R. Webster, K. Y. Volyanskyy, and M. J. Weissburg, "Bioinspired algorithm for autonomous sensor-driven guidance in turbulent chemical plumes," Bioinspiration \& Biomimetics, vol. 7, no. 3, p. 036023, 2012.

[16] S. Edwards, A. J. Rutkowski, R. D. Quinn, and M. A. Willis, "Mothinspired plume tracking strategies in three-dimensions," in Proc. 2005 IEEE Int. Conf. Robotics and Automation, 2005, pp. 1669-1674.

[17] W. Li, J. A. Farrell, S. Pang, and R. M. Arrieta, "Moth-inspired chemical plume tracing on an autonomous underwater vehicle," IEEE Transactions on Robotics, vol. 22, no. 2, pp. 292-307, 2006.

[18] P. Pyk, S. B. i. Badia, U. Bernardet, P. Knsel, M. Carlsson, J. Gu, E. Chanie, B. S. Hansson, T. C. Pearce, and P. F. M. J. Verschure, "An artificial moth: Chemical source localization using a robot based neuronal model of moth optomotor anemotactic search," Autonomous Robots, vol. 20, no. 3, pp. 197-213, 2006.

[19] N. Lemmens, S. de Jong, K. Tuyls, and A. Nowé, "Bee behaviour in multi-agent systems," in Adaptive Agents and Multi-Agent Systems III. Adaption and Multi-Agent Learning., ser. Lecture Notes in Computer Science. Springer, 2008, vol. 4865, pp. 145-156.

[20] A. Berdahl, C. J. Torney, C. C. Ioannou, J. J. Faria, and I. D. Couzin, "Emergent sensing of complex environments by mobile animal groups," Science, vol. 339, pp. 574-176, 2013.

[21] W. Wu, I. D. Couzin, and F. Zhang, "Bio-inspired source seeking with no explicit gradient estimation," in 3rd IFAC Workshop on Distributed Estimation and Control in Networked System, 2012, pp. 240-245.

[22] D. R. Webster, K. Y. Volyanskyy, and M. J. Weissburg, "Sensorymediated tracking behavior in turbulent chemical plumes," in 7th International Symposium on Turbulence and Shear Flow Phenomena, Ottawa, Canada, 2011.

[23] H. K. Khalil, Nonlinear Systems, 3rd ed. Prentice Hall, 2002. 